\title{
A User-Study Examining Visualization of Lifelogs
}

\author{
Soumyadeb Chowdhury \\ Singapore Institute of Technology \\ Soum.Chowdhury@singaporetech.edu
}

\author{
Md Sadek Ferdous \\ University of Southampton \\ S.Ferdous@soton.ac.uk
}

\author{
Joemon M Jose \\ University of Glasgow, \\ Joemon.Jose@glasgow.ac.uk
}

Abstract - With continuous advances in the pervasive sensing and lifelogging technologies for the quantified self, users now can record their daily life activities automatically and seamlessly. In the existing lifelogging research, visualization techniques for presenting the lifelogs and evaluating the effectiveness of such techniques from a lifelogger's perspective has not been adequately studied. In this paper, we investigate the effectiveness of four distinct visualization techniques for exploring the lifelogs, which were collected by 22 lifeloggers who volunteered to use a wearable camera and a GPS device simultaneously, for a period of 3 days. Based on a user study with these 22 lifeloggers, which required them to browse through their personal lifelogs, we seek to identify the most effective visualization technique. Our results suggest various ways to augment and improve the visualization of personal lifelogs to enrich the quality of user experience and making lifelogging tools more engaging. We also propose a new visualization feature- drill-down approach with details-ondemand, to make the lifelogging visualization process more meaningful and informative to the lifeloggers.

\section{INTRODUCTION}

With several wearable cameras recently introduced in the commercial market, such as Autographer [1] and Narrative Clip, lifelogging has started to gain mainstream attraction, and has become a normative activity. These cameras can passively capture thousands of images throughout the day, without the need of explicit interaction due to their hands-free nature. Due to the vast amount of images captured, the challenges include, but are not limited to structuring, organizing, summarizing, searching through and visualizing, increasing number of lifelog images in a meaningful way [2]. Despite the growing number of studies using lifelogging such as with wearable cameras, there has been little consideration on how to visualize and present the lifelogs in a user-friendly yet meaningful way. A handful of the existing works [3, 4] have proposed a number of design guidelines and visualization techniques to present the lifelog images, but they have not reported any user studies to evaluate the effectiveness of the proposed techniques. It is our conjecture that visualization of lifelog images is a necessary aspect, which must be considered, when designing lifelogging tools to enrich the user experience and support their needs. This paper will present a user study which was conducted with 22 lifeloggers, who had used two devices (Autographer, which is a wearable camera and a GPS device) for a period of three days to investigate the effectiveness of four different interaction metaphors (visualization techniques). The key research question investigated in the study is, R1: How can we effectively aggregate and visualize the lifelogs obtained from a wearable camera as well as a GPS tracker (two distinct devices used

978-1-4673-8695-1/16/\$31.00@2016 IEEE simultaneously) and present them to a lifelogger ( $L L)$, in a meaningful way? We seek to compare the effectiveness of the following four visualization techniques from a lifelogger's perspective: (1) temporal; (2) tempo-spatial; (3) tempo-spatialvisual; (4) most visited locations. Additionally, we will explore, R2: whether lifeloggers' (LLs') user experience can be improved by recommending nearby places of interest to the locations which are frequently visited by them. This feature will provide customized information to a LL based upon their lifelogs, to make the visualization engaging and informative. We seek to understand the potential of such a feature.

\section{BACKGROUND WORK}

Gurrin et al. [5] have provided a comprehensive review of the lifelogging research and have argued that the most common proposition is, by capturing data about our daily activities, such systems tend to offer effective support for recollecting and remembering our past. However, lifelogging research with regards to topics in user interaction, in particular, applying visualization as a means of abstracting and summarizing information is sparse. To the best of our knowledge none of the existing works have reported user studies to either demonstrate the effectiveness of one or more visualization metaphors. Most of the existing studies have focused on visualizing data obtained from the activity trackers such as Fitbit [6], and Pedometer, primarily focusing on numerical fitness data (such as tracking number of steps covered in a day, distance walked etc.), which are presented using charts, figures etc. However, the lifelogs generated from multiple sensor sources (Autographer and GPS) are more media-rich (for e.g. vast amount of visual data may need to be aligned to GPS data) compared to the numerical fitness data, and requires careful consideration on how to present and summarize such information. In relation to the user interaction in lifelogging, Hopfgartner et al. [3] have outlined various design templates that may help to support different visualization schemes and perhaps invoke further research efforts in this direction. The authors have suggested that visual diaries should summarize a user's day, a comic-book style interface should be used when the screen space is limited (handheld devices) and a master-detailed interface should be used to facilitate lifelog image exploration. The authors not reported any user studies to demonstrate the effectiveness of the aforementioned techniques. Meyer et al. [4] have also proposed a number of design guidelines, but for the data obtained from the activity trackers. Based on a study conducted with 12 users, who were required to use a number of trackers, the authors suggested that: (1) A detailed visualization (showing all the data) is useful for a user in the first instance, but after some time (period of use) the users would look for limited information, which needs to be 
presented instantaneously. Hence the interface should support both types of visualization, so that the users can choose the appropriate interaction method, depending upon their needs and interests; (2) Users are more interested in the reliability of the visualization and showed discontent, if the data did not reflect their behavior correctly. In the author's words "fuzzy but reliable data is better than pseudo-precise but possibly wrong data". Hence it is essential to present the data accurately to the user for improving the user experience.

We contend that the visualization techniques will depend upon the preferences of a user, needs of the user, context of use, the types and quantity of the lifelogs. We believe the lifelog content is media-rich, and different products representing different paradigms require different solution. Since there is no known single best way to aggregate and visualize lifelogs obtained from an image capturing device and a GPS tracker, we present a lifelogging web-based application which is used as the platform to conduct a user study for systematically assessing four visualization techniques. The results obtained from the user study will contribute towards lifelogging HCI (user-centric) research, by understanding the effectiveness and limitations of the visualization techniques presented in this paper from the perspectives of the LLs.

\section{LIFELOGGING WEB APPLICATION}

We have developed a Lifelogging Web Application (LWA), which enables the LLs to upload their lifelog images and GPS locations, followed by reviewing their images and deleting them (as appropriate), before the images are stored in our back-end for visualization. Prior to developing the LWA, we had used both the devices for a week, to form a collection comprising of 18234 images and 97654 GPS coordinates (data-set1), which was used to test the different visualization modules in LWA, before conducting the user evaluation. The description of each module is discussed below.

\section{A. Temporal Clustering (T1)}

The LLs can view their lifelog images through a visual image diary and GPS logs using a trails diary (Fig 1a), in two separate interfaces for a range of dates, which can be selected using a date-picker. This module employed temporal clustering (T1) to display the lifelogs, using the lifelog timestamp.

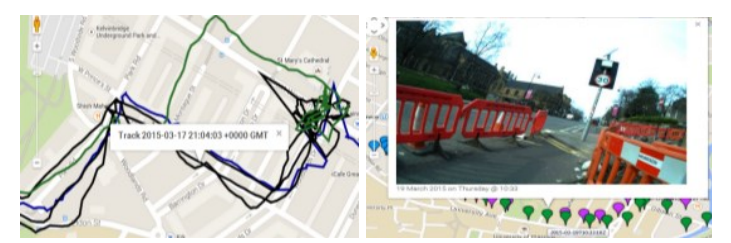

Fig. 1. (left to right) a. Trails diary; b. Image-Location mapping

\section{B. Tempo-Spatial Clustering (T2)}

This module assigned each image (from the Autographer) to a GPS location (obtained from the GPS device), using the timestamp as the index. The tempo-spatial clustering is employed one of the design guideline proposed by Meyer et al. in [4], i.e. providing more information about the lifelogs compared to $\mathrm{T} 1$, by making the lifeloggers aware of the location where the images are captured. The resultant output is displayed as markers corresponding to the location of an image on a map, with a marker window containing the image and the timestamp (Fig 1b). Each day/date is shown using a distinct colored marker. Our approach takes the timestamp of an image (tsimage) as the input, and then finds a corresponding match first for the range (tsimage -5 to $\left.\mathrm{ts}_{\text {image }}+5\right)$ and then (tsimage -10 to tsimage +10 ) from the GPS logs. The parameter ( $\mathrm{ts}_{\text {image }} \pm x$ seconds) was determined using dataset- 1 . We found that: $80 \%$ of the images were assigned a location for the range ( $x=5$ seconds); $86 \%$ of the images were assigned a location for the range ( $x=10$ seconds). A location was not found for $14 \%$ to $20 \%$ of the images because the GPS device could not log the locations, while the LL was indoors, i.e. inside the buildings
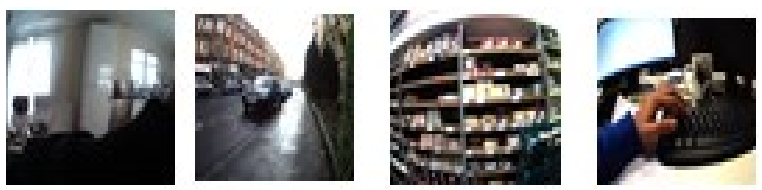

Fig. 2. Visual Summary comprising of 4 images

\section{Tempo-Spatial-Visual Clustering (Visual Summary, T3)}

This module displays the visual summary (Fig 2) of a lifelogger's day (i.e. key moments), by clustering the images based on temporal, spatial and visual information. The visual summary was generated using the approach proposed in [7]: (1) Eliminating blurred images using a Haar wavelet transform [8]; (2) Eliminating visually similar images using pHash [9], and clustering them together using hamming distance; (3) Modelling the images as a $515 \mathrm{D}$ vector (modelling the images as 3-D vector of its time and location, and concatenating it with 512-D vector extracted from the GIST features [10] for the image). Finally, Expectation-maximization algorithm is employed to form the summaries. The visual summary comprising of 20-40 images (for each day) is displayed using an interactive carousel to the user.

\section{Most Frequently Visted Locations (Trending Locs, T4)}

The trending locations are a collection of maximum eight geo-coordinates that a LL has either frequently visited, or has spent most of their time, based upon the logs captured by the GPS device. The trending locations are visualized on a heat map generated using all the location logs uploaded by a LL as Yellow markers having a star icon (corresponding to the coordinates), containing an image captured in the location with the street name (Fig 3a). This module is inspired by one of the guidelines proposed in [4], which suggests that limited information (a meaningful summary) corresponding to the user's vast data should be presented instantaneously. The steps used to generate the trending locations are: Step 1: All the location coordinate pairs are taken as input, and formatted upto 4 places of decimal and the frequency of the unique pairs (which represents popularity) calculated, before sorting them in the decreasing order of popularity. We used the data-set1 and empirically found that an accuracy of $(11 \mathrm{~m})$, i.e. 4 decimal places will be able to distinguish between the location of a lifelogger accurately; Step 2: The top 100 pairs are chosen (as opposed to average popularity to avoid bias of the 
popularity distribution, and total number of points) and the distance (d) between two location pairs is calculated using the Haversian equation [11]. If the distance between the two pairs $(\mathrm{d}<600 \mathrm{~m})$, the pair with the higher popularity replaces the other; Step 3: The street names of each pair (in Step 2) are extracted using the Geocode Farm API [12]. The unique names are clustered and the top 8 locations based upon the popularity is the final output. The results using the data-set1 showed that the trending locations are likely to point over the densely populated regions in the heat map (red zones, Fig 3b).

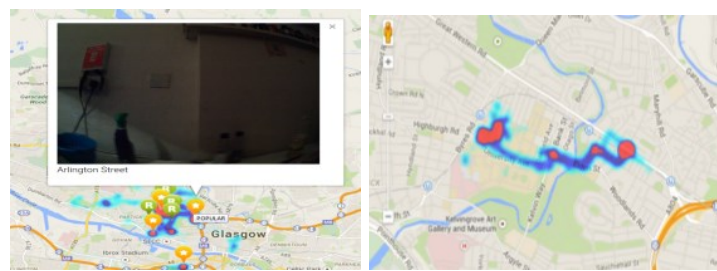

Fig. 3. (left to right) a. Trending Locs and Recommendations; b. Heat Map

\section{E. Near-by Places of Interest (Recommendations)}

A maximum of 16 places of interest nearby to the trending locations are displayed in the same heat-map as the T4. The recommendations are meant to make the LLs aware of the interesting places near the trending locations. We hypothesize that this feature in addition to the summaries (T4) may make the LWA interesting and engaging to use for personal benefits. The following steps were followed to generate the list of recommended places: Step 1: The trending location coordinates are extracted and then each one of them is sent to the Google Places API [13], which returns the 20 nearby places (within $500 \mathrm{~m}$ radius) for each location. An additional optional parameter, 'type', is used to specify the different categories of places (e.g. museum, nightclub art-gallery, café etc.), to restrict the results only to the selected categories; Step 2: The frequency of the occurrence for each place (in Step 1) is calculated and a list of unique places is generated. The top 10 places based upon the popularity are visualized on the map as green markers (having an icon R), corresponding to the location, containing the place name and address (Fig 3b). If all the places have the same popularity, two places are selected randomly for each trending location, i.e. 16 in total.

\section{USER STUDY}

The objective of the experiment is to investigate the effectiveness of four visualization techniques for exploring lifelog data collected by lifeloggers. We recruited 22 subjects by sending emails to the university mailing list. These subjects agreed to voluntarily take part in our experiment, and use lifelogging devices (wearable camera Autographer and GPS device) for a period of 3 days, as well as share their data with us for the purpose of research only. A pre-study questionnaire was used to collect the demographic information (Table 1) of the subjects, and also confirmed that they have not used lifelogging devices in the past. This study was approved by the ethics committee of the institution. A three-stage study framework was designed to fulfill the objectives of the experiment, which is discussed below.

TABLE I.
\begin{tabular}{|l|l|}
\hline Gender & Female -8; Male -14 \\
\hline Profession & Student-14; Researcher-5; Staff:3 \\
\hline Age Range & 21-40; Mean: 26; SD: 4.92 \\
\hline Time spent in the city & $\begin{array}{l}\text { upto 6 months: 5; upto 1 yr: 5; } \\
\text { more than a yr:12 }\end{array}$ \\
\hline
\end{tabular}

\section{A. Stage 1-Data Gathering}

The LLs (subjects) were asked to use both the devices simultaneously, for a period of 3 days (6-8 hours every day). The devices were given for a week, to ensure some slack time and ensuring the LLs get used to the devices. The time frame was limited due to the voluntary nature of the participation, and to avoid subject attrition. LLs were provided with an instruction sheet to help them use the devices, which was also demonstrated in person, followed by a practice session. Additionally, a list of suggestions were provided in order to reduce the potential risks arising from our study. The suggestions included, but were not limited to: (1) avoiding wearing the device in rest rooms; (2) avoiding wearing the device without the consent of others in situations where people expect privacy (e.g. common areas in shared flat, workplace), and places where photography may be prohibited; (3) if a bystander enquired about the device, first the image capture must be paused, and then the objectives of our study must be explained; (4) if a bystander seems to be discontent or concerned, then step 3 should be followed and they should be provided with a business card containing our contact information, as well as a URL containing further details about our project. The participants were also asked to note down the date and time of the incident in a deletion card, so that we could delete the images for that period of time. This framework ensured that the study reflected the way these devices will be actually used in real-life (LLs environment).

\section{B. Stage 2- Exploring Lifelogs}

The LLs were asked to meet the evaluator in person and then upload the lifelogs, review them (delete images as appropriate) and finally confirm their submission to LWA. Once the lifelogs were uploaded, LLs were asked to answer a questionnaire (Q1) capturing information regarding: (Q1.1) reasons for reviewing the images; $(\mathbf{Q} 1.2)$ reasons for pausing the image capture; (Q1.3) incidents that happened while using the device. Next, they were required to use each visualization feature in LWA. The main tasks comprised of: viewing the image and trail diary (T1); using the image to location viewer (T2); exploring the visual summaries (T3); exploring the trending locations (T4); finding out interesting interest nearby to the trending locations (T5).

\section{Stage 3- Post Study Questionnaire}

A face-to-face interview was conducted with the LLs. The interview questions evaluated the effectiveness, and visual appeal of T1-T4, in addition to the most preferred technique. Visual aesthetics was evaluated because it is believed to have a substantial effect on impression judgements [14]. The other aspects (effectiveness, and preference) which are defined by the international standard ISO/IEC 9241-11 [15], have been extensively used to evaluate user interfaces. The subjective feedback provided by the lifeloggers was also captured. 


\section{RESULTS FOR QUESTIONNAIRE (Q1)}

\section{A. Data Collection}

Table II shows the statistics of the lifelogs captured by 22 LLs. The number of images containing locations is $89 \%$ because the GPS device did not log the locations, once the lifelogger went in to an indoor location, i.e. supermarket.

TABLE II. LIFELOG COLLECTION STATISTICS
\begin{tabular}{|l|l|l|}
\hline & \multicolumn{2}{|l}{ Image Num Comments } \\
\hline Images Captured (Ic) & 21256 & Autographer \\
\hline Images deleted (Id) & 2765 & 22 lifeloggers \\
\hline Images stored (Is) & 18491 & (Ic-Id) \\
\hline Coordinate points (Cp) & 99015 & GPS logs \\
\hline Images with location & 16457 & (Is $\times 89) / 100$ \\
\hline Visual duplicates (Iv) & 7899 & $($ Is $\times 48) / 100$ \\
\hline Poor quality & 6583 & (Is $\times 35.6) / 100$ \\
\hline
\end{tabular}

\section{B. Q1.1-Reviewing images}

All the LLs reviewed their images, before storing them in our web-app. The primary reasons for reviewing are, but not limited to: (1) curiosity regarding the images captured; (2) eliminating personal images; (3) examining the images to eliminate sensitive information captured during the usage, like logging into a bank account. The LLs also felt that the reviewing process was time-consuming, as they had to browse through many images including the ones with poor quality.

\section{Q1.2- Pausing the image capture}

All the LLs reported to have paused capturing the images on a number of occasions. The responses revealed that most LLs: exerted a high degree of physical discipline, i.e. avoided capturing photos of private moments, official meetings etc.; were concerned about impression management, i.e. avoided capturing photos of their generic habits (room cleanliness); avoided capturing private information, i.e. emails on computer screen and while using ATM machines. These results revealed that the LLs in our study were cautious, while using the device, and refrained from capturing images in scenarios, which they perceived as sensitive and private.

\section{Q1.3: Anecdotes while using the device}

The responses revealed that there were a number of occasions ( 5 in total), where the LLs were asked by an unknown person or a known associate (friends, flat mates, colleagues) to stop using the camera. On two of the occasions (unknown people in public transport - bystanders), enquired about the device, and showed discontent after hearing its characteristics. The bystanders requested the LLs to delete their photographs, because they did not feel comfortable being photographed randomly. In two other occasions the LLs were asked either by their partners or flat mates to refrain from using the device indoors, because the latter felt that the images captured by the device are a breach of their personal privacy, especially inside the shared flats. There was one instance, when the line manager (workplace) asked the LL to avoid using the camera during office hours, and in business gatherings, though there were no organization policies to stop people from taking pictures (according to the LL). These findings raise serious privacy concerns (from a bystander's perspective), as well as the social acceptability of the technology in the real-life. This paper did not examine the privacy implications in lifelogging.

\section{RESULTS FOR POST-STUDY QUESTIONNAIRE (Q1)}

\section{A. Effectiveness}

We seek to understand the effectiveness of the four techniques (T1-T4) to present the lifelogs. This aspect is further segregated into the following four dimensions based upon the questionnaires, USE [16], MUMMS [17] and ASQ [18], to better collate the LLs' perspectives. (A1) how useful is the presented information; (A2) how meaningful is the presented information; (A3) is the information presented clearly and can be easily interpreted, without the need of any additional expertise; (A4) Does the visualization help the LLs in exploring the lifelogs in a productive way. LLs were asked to rate each technique on a scale of 1 to 5 (1 being the least score and 5 being the highest), for all the dimensions (A1-A4). The mean scores for each dimension in addition to the mean effectiveness score is presented in Fig 4.

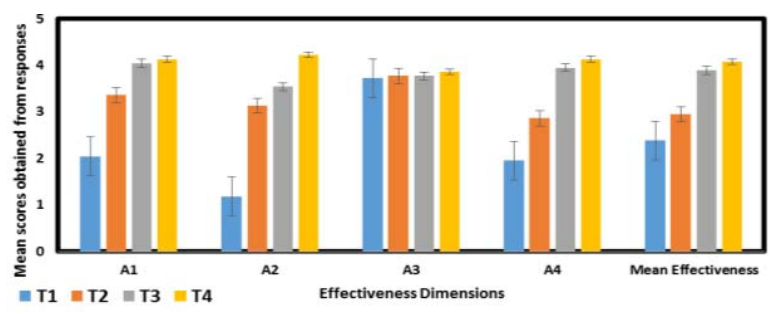

Fig. 4. Mean scores for Effectivenss

A Wilcoxon test (post-hoc applying a Bonferroni correction), showed significant differences $(p<0.008)$ in the mean effectiveness scores for all the pairs except between T3 (visual summary) and T4 (trending locations), suggesting that T3 and T4 were rated equally effective. Further analysis shows that T4 was rated the best in terms of usefulness (A1), meaningfulness (A2), and exploring the lifelogs (A4). The comments from the LLs highlighted that T4 summarizes the lifelogs in a meaningful way without increasing the information overload, i.e. displayed the most visited locations with the corresponding images, which LLs also perceive as useful. Additionally, the LLs pointed out that the technique decreases the cognitive load, because even if the amount of lifelogs increase, limited amount of information is displayed, which makes it easy to explore the lifelogs, compared to other techniques. Moreover, in relation to T3 (visual summary), LLs felt it is certainly useful to view the day in images, but the lack of location information makes it less useful compared to T4. Moreover, the number of images increases, when the LLs search across a range of dates, which makes exploring the lifelogs a bit time-consuming and less productive compared to T4. The mean scores $(\simeq 4)$ in relation to the dimension A3, demonstrated that all the LLs were satisfied and were able to understand the information presented using all the techniques (T1-T4), equally well. 


\section{B. Visual Aesthetics}

In terms of visual aesthetics, the LLs were again asked to rate the techniques (T1-T4) on scale of 1 to 5 ( 1 being least visually appealing and 5 being highly appealing). The mean scores showed that T4 (trending locations) had the highest visual appeal followed by T2 (tempo-spatial clustering), then T3 (visual summary), and finally $\mathrm{T} 1$ (temporal clustering). Based upon the subjective comments of the LLs, visualizing the image information in the maps enhanced the look and feel feature, and the interactivity. The LLs suggested that the visual appeal is likely to improve for T2 and T4, by displaying the images in a separate carousel below the map. Moreover, the LLs felt that T2 and T4 were more colorful due to the presence of markers and also provided them with additional location information (compared to T1 and T3).

\section{User Preferences}

Using this aspect, we seek to understand the visualization technique which was most preferred by the LLs. They were asked to rate each technique on a scale of 1 to 5 ( 1 being the least preferred and 5 being the most preferred). The ratings corresponding to each technique are shown in Fig 5

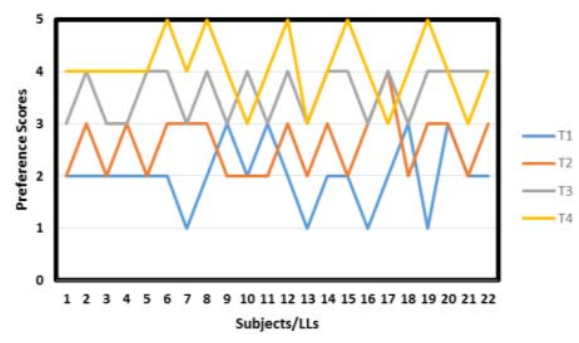

Fig. 5. Scores for user preferences

A Wilcoxon test (applying a Bonferroni correction), showed that the ratings were significant $(p<0.008)$ for all the pairs, except between $\mathrm{T} 1$ and T2. Hence according to the Figure 5 and the statistical tests, T4, i.e. displaying the trending locations was the most preferred technique followed by T3 (visual summary), then T2 and finally T1.

\section{Recommendation feature}

In the context of the recommendation feature implemented in LWA, the LLs were asked to rate each of the following aspects (K1-K4) on a scale of 1 to 5 (1 being the least score and 5 being the highest): (K1) Whether the customized feature made the lifelog exploration engaging; (K2) Whether the feature provided useful information in addition to the summary; (K3) Whether the feature helped the LLs gain valuable insights about the location surrounding the trending locations; (K4) Whether the feature enhanced the user experience, i.e. LLs felt satisfied with this information in addition to the lifelogs. The scores given by the LLs for each aspect is presented in Fig 6. The results showed that the mean scores for all the aspects were $(>4)$, which suggests that the LLs were very satisfied with the feature, and found it to be very engaging $($ mean $=4.1)$ useful $($ mean $=4.1)$ and informative (mean=4.1), in addition to the lifelog trending locations summary. Moreover, the mean score for R4 (=4.65) clearly suggests that the feature enhanced the user experience, and made the lifelog exploration engaging and informative, by providing relevant customized information (nearby places of interest) using external resources (Google Places API.

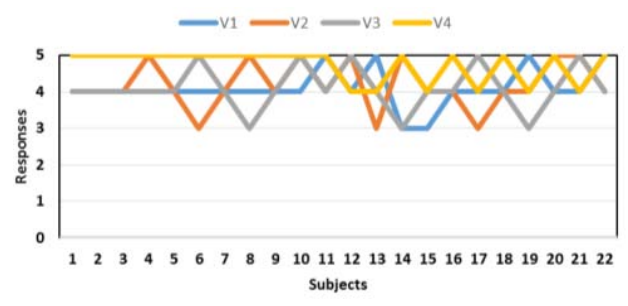

Fig. 6. Scores for Recommedation Feature

\section{DISCUSSION}

To answer the key research question (R1), this paper examined four visualization techniques. The results demonstrated that presenting the frequently visited locations (technique-T4) with the corresponding image, timestamp and the location name on a map was the most effective representation to aggregate and visualize the lifelogs, and was also more visually appealing as well as preferred by the LLs, compared to the other techniques. This is likely attributed to the: (a) meaningful abstraction of the lifelogs by displaying the most frequently visited locations with additional information, which the LLs perceived as useful; (b) high visual aesthetics of the interface using the map and components like colorful markers, which in-turn enhanced interactivity; (c) facilitating the LLs to explore the vast archives in a productive manner by decreasing the information and cognitive overload, i.e. even when the archive size increases, the visualization displayed a meaningful summary, which makes it less time-consuming to use. In the words of a LL, "I was very satisfied with the simple, yet informative summarized view which reflected my data accurately".

In relation to $\boldsymbol{R} 2$, i.e. making lifelogging exploration more engaging and informative, the potential of the feature recommending places of interest near to the trending locations, (using Google Places API) was examined. The results demonstrated that the feature was perceived to be very useful and meaningful by the LLs, and enhanced the user experience. Given the proliferation of the data that can be gathered using publicly available resources and APIs (news websites, twitter, and Flickr), we can develop more customized features and visualize them in lifelogging apps to make the LLs more aware of their surroundings and facilitate discovery of new content, which would further enrich the LLs' multimedia quality of experience (QoE).

Based upon our findings, we propose to incorporate a master-detailed design using a drill-down approach to further extend the features offered by the trending location (T4). The aforementioned design is based upon Shneiderman's philosophy [19] which suggests that visualization should represent the abstract information in a meaningful way to facilitate further exploration. Our proposal aims to first provide the LLs a meaningful overview using trending location markers on a heat map. This is followed by details on demand (drill-down), i.e. display more images from the surrounding location, which could be selected by the LLs 
using a bounding box on the map. The images will be displayed in two separate carousels: (1) displaying the images from the lifelog archive of the LL; (2) displaying images obtained from publicly available sources (twitter, Instagram and Flickr). The proposed design will help the LLs explore increasing number of lifelogs in a single interface, and the additional image carousel (2) is more likely to improve the user engagement and experience. Examining the proposed design with LLs will help us to discover further ways to increase LLs engagement and cater to their growing needs.

\section{CONCLUSION}

This paper contributes towards lifelogging HCI by evaluating four distinct visualization techniques developed using state-of-the art components to explore personal lifelogs, in addition to examining a novel feature meant to make the visualization engaging and informative. The results discussed in the previous section suggests that the meaningful and useful summarization of the lifelogs using technique T4 (trending locations), in visually appealing and informative interfaces was the most preferred technique by the LLs, and the additional feature which recommended nearby places of interest improved the user engagement and experience, while exploring personal lifelogs. These results suggests that the visualization technique employed in lifelogging applications should be: insightful, i.e. the information presented to the LLs while exploring the lifelogs is useful and made meaningful (by generating a sensible summary of the vast archive); intuitive i.e. LLs can explore the lifelogs and interpret the results of the visualization easily, without the need of any additional expertise; interactive, i.e. a number of interface components are provided to the LLs to view an array of information; impressive, i.e. the visualization is aesthetically attractive to improve the user experience; immersive, i.e. novel features should be included to enrich user experience by facilitating the discovery of new content beyond the personal archives and increased awareness about the surroundings, using publicly available resources. Though the subject sample in our study was small, and the data collection was limited to 3 days, but our study is the first of its kind which focusses on lifelogging visualization, user engagement, QoE, and provides valuable clues for designing lifelogging visualization tools in the future, incorporating user-centric design. Based upon our preliminary findings, we contribute to the field of lifelogging research by proposing a possible metrics, i.e. the five 'Is' (insightful, intuitive, interactive, impressive and immersive), which could be likely used in the future for evaluating lifelogging visualization mechanisms. Additionally, using the metrics consistently across all the future user studies involving lifelogging visualization and interaction techniques, is likely to facilitate benchmarking and compare different techniques.

\section{ACKNOWLEDGEMENT}

The authors acknowledge support from Integrated Multimedia City Data (iMCD), a project within the ESRC-funded Urban Big Data Centre (ES/L011921/1).

\section{REFERENCES}

[1] Autographer, "The world's First intelligent Wearable camera. Clip-on, go out," Autographer. [Online]. Available: http://www.autographer.com. Accessed: Feb. 14, 2016.

[2] Melucci Massimo. "Contextual Search: A Computational Framework."Foundations and Trends in Information Retrieval 6, no. 4-5 (2012): 257-405

[3] Frank Hopfgartner Yang Yang, Lijuan Marissa Zhou, and Cathal Gurrin. "User interaction templates for the design of lifelogging systems." InSemantic Models for Adaptive Interactive Systems, pp. 187-204. Springer London, 2013.

[4] Jochen Meyer, Jutta Fortmann, Merlin Wasmann, and Wilko Heuten. "Making lifelogging usable: Design guidelines for activity trackers." InMultimedia Modeling, pp. 323-334. Springer International Publishing, 2015.

[5] Cathal Gurrin, Alan F. Smeaton, and Aiden R. Doherty. "Lifelogging: Personal big data." Foundations and trends in information retrieval 8, no. 1 (2014): 1-125.

[6] Fitbit, "Fitbit official site for activity Trackers \& more," [Online]. Available: https://www.fitbit.com/. Accessed: Feb. 14, 2016.

[7] Soumyadeb Chowdhury, Philip J. McParlane, Md Sadek Ferdous, and Joemon Jose. "My day in review: Visually summarising noisy lifelog data." In Proceedings of the 5th ACM on International Conference on Multimedia Retrieval, pp. 607-610. ACM, 2015.

[8] Hanghang Tong, Mingjing Li, Hongjiang Zhang, and Changshui Zhang. "Blur detection for digital images using wavelet transform." In Multimedia and Expo, 2004. ICME'04. 2004 IEEE International Conference on, vol. 1, pp. 17-20. IEEE, 2004.

[9] Alexander Hauptmann, Rong Yan, and Wei-Hao Lin. "How many high-level concepts will fill the semantic gap in news video retrieval?" In Proceedings of the 6th ACM international conference on Image and video retrieval, pp. 627-634. ACM, 2007.

[10] Aude Olivia, and Antonio Torralba. "Building the gist of a scene: The role of global image features in recognition." Progress in brain research 155 (2006): 23-36.

[11] Chris Veness "Calculate distance and bearing between two Latitude/Longitude points using Haversine formula in JavaScript." Movable Type Scripts (2011).

[12] Geocode Farm, "Free Geocoding API,". [Online]. Available: https://www.geocode.farm. Accessed: Feb. 14, 2016.

[13] Google Places, "Place types," Google Developers, 2016. [Online]. Available:https://developers.google.com/places/supported_types. Accessed: Feb. 14, 2016.

[14] Gitte Lindgaard, Cathy Dudek, Devjani Sen, Livia Sumegi, and Patrick Noonan. "An exploration of relations between visual appeal, trustworthiness and perceived usability of homepages." ACM Transactions on Computer-Human Interaction (TOCHI) 18, no. 1 (2011): 1.

[15] Ben Shneiderman. Designing the user interface. Pearson Education India, 2003.

[16] Arnold M Lund. "Measuring usability with the USE questionnaire." Usability interface 8, no. 2 (2001): 3-6.

[17] Sung Heum Lee. "Usability testing for developing effective interactive multimedia software: Concepts, dimensions, and procedures." Educational Technology \& Society 2, no. 2 (1999): 1-13.

[18] James R Lewis. "IBM computer usability satisfaction questionnaires: psychometric evaluation and instructions for use." International Journal of Human-Computer Interaction 7, no. 1 (1995): 57-78.

[19] Ben Shneiderman. "The eyes have it: A task by data type taxonomy for information visualizations." Visual Languages, 1996. Proceedings. IEEE Symposium on. IEEE, 1996, 336-34 